\title{
Review Article \\ Importance of Molds for Nanoimprint Lithography: Hard, Soft, and Hybrid Molds
}

\author{
B. Kwon ${ }^{1}$ and Jong H. Kim ${ }^{2,3}$ \\ ${ }^{1}$ Department of Materials Science and Engineering, University of Washington, Seattle, WA 98195, USA \\ ${ }^{2}$ Department of Chemical Engineering Education, Chungnam National University, 99 Daehak-ro, Yuseong-gu, \\ Daejeon 305-764, Republic of Korea \\ ${ }^{3}$ Graduate School of Energy Science and Technology, Chungnam National University, 99 Daehak-ro, Yuseong-gu, \\ Daejeon 305-764, Republic of Korea
}

Correspondence should be addressed to Jong H. Kim; jonghkim@cnu.ac.kr

Received 30 March 2016; Accepted 24 May 2016

Academic Editor: Tian Xia

Copyright (C) 2016 B. Kwon and J. H. Kim. This is an open access article distributed under the Creative Commons Attribution License, which permits unrestricted use, distribution, and reproduction in any medium, provided the original work is properly cited.

\begin{abstract}
Nanoimprint lithography has attracted considerable attention in academic and industrial fields as one of the most prominent lithographic techniques for the fabrication of the nanoscale devices. Effectively controllable shapes of fabricated elements, extremely high resolution, and cost-effectiveness of this especial lithographic system have shown unlimited potential to be utilized for practical applications. In the past decade, many different lithographic techniques have been developed such as electron beam lithography, photolithography, and nanoimprint lithography. Among them, nanoimprint lithography has proven to have not only various advantages that other lithographic techniques have but also potential to minimize the limitations of current lithographic techniques. In this review, we summarize current lithography techniques and, furthermore, investigate the nanoimprint lithography in detail in particular focusing on the types of molds. Nanoimprint lithography can be categorized into three different techniques (hardmold, soft-mold, and hybrid nanoimprint) depending upon the molds for imprint with different advantages and disadvantages. With numerous studies and improvements, nanoimprint lithography has shown great potential which maximizes its effectiveness in patterning by minimizing its limitations. This technique will surely be the next generation lithographic technique which will open the new paradigm for the patterning and fabrication in nanoscale devices in industry.
\end{abstract}

\section{Introduction}

Nanoscale fabrication method with high resolution and large yield has been the prominent research area due to its crucial role in patterning the nanosized devices in array form for various applications, ranging from electronic memory application to biomedical application [1-15]. For these applications, there are two main different fabrication methods: "bottomup" chemical method and "top-down" lithographic method. Conventionally, "bottom-up" chemical synthesis method has been used to fabricate simple metal/oxides particles [1626], polymer elements [27-32], or self-assembled elements [33-37]. These methods demonstrate resulting devices with decent size control, monodispersity, and large production scale $[19,38,39]$. However, they posed critical difficulties of controlling the shape, size, structure, and defects of resultant devices. To solve such difficulties, physical "topdown" lithographic methods have been proposed [1]. These physical "top-down" lithographic methods are including, but not limited to, electron beam lithography, photolithography, and nanoimprint lithography. They have shown the great potentials for patterning materials into nanostructures, especially into ordered arrays. In order to further improve the quality of the fabricated elements, different lithographic techniques have been also proposed for different purposes.

Electron beam lithography is a technique which uses the electron beam to pattern element's arrays. Specifically, the focused beam of electrons is used to draw custom shapes on a surface covered with an electron-sensitive film called a resist [40-43]. As an electron beam is exposed to the specific part 
of the resist, electron beam changes the solubility of the resist, enabling selective removal of either the exposed or nonexposed regions of the resist by immersing the film into the developer. As resolution of focused electron beam can be precisely controlled, electron beam lithography typically demonstrates $<10 \mathrm{~nm}$ resolution [41-45]. However, it posed critical difficulties since the focused electron beam cannot expose multiple elements simultaneously, which causes extensive writing time and high-cost issue. Compared to electron beam lithography, photolithography, also called optical lithography or UV lithography, is a simple and cost-effective patterning method. It uses light to transfer a geometric pattern from a photomask to a light-sensitive chemical "photoresist" on the substrate, which changes the solubility of the exposed resist. Depending on the purpose of lithography, two different kinds of photoresist can be used: positive-tone photoresist and negative-tone photoresists. The fundamental difference between these two photoresists is the solubility change when exposed to the light: the solubility of exposed resist is faster (positive-tone photoresist) or slower (negative-tone photoresist). Even though the photolithography can be a simple largearea patterning method with low-cost, the critical limitation still exists due to the wavelength of the UV-light, which ultimately restricts sub-100 $\mathrm{nm}$ scale patterning $[46,47]$.

In order to solve both technical limitations of electron beam lithography and photolithography, nanoimprint lithography was first proposed by Chou et al. [4,5]. Nanoimprint lithography creates the patterns by mechanical deformation of imprint resist and subsequent processes. Earliest form of nanoimprint lithography proposed by Chou et al. is based on thermoplastic polymers as resist materials $[4,5]$. In standard thermoplastic nanoimprint lithography, a thin layer of imprint resist is spin-coated on the sample substrate. Then the mold, which was predefined with topological patterns, is pressed onto the resist-coated substrate with increased temperature (above glass transition temperature $\left(T_{\mathrm{g}}\right)$ ) and elevated pressure. The pattern on the mold is transferred onto the softened resist, and after being cooled down, the resist hardens. After the mold is separated from the sample, then patterned resist remains on the substrate. Since the initial idea of this technique was developed, numerous variations of the nanoimprint lithography have been suggested. Specifically, the photo-nanoimprint lithography has been proposed in order to substitute required high temperature to soften the thermoplastic resist into UV-light to harden the resist to transfer the pattern $[3,48-52]$. Based on these two hardening methods, three different types of nanoimprint lithography methods have been suggested in order to achieve high resolution, large-area, and low-cost patterning: soft-mold nanoimprint lithography, hard-mold nanoimprint lithography, and hybrid-mold nanoimprint lithography.

In this review paper, we will discuss three types of nanoimprint lithography methods which are based on different moduli of molds: soft mold, hard mold, and hybrid mold. We will first summarize recent research progresses in each method; then we will further highlight the advantages and disadvantages of them. Finally, we will suggest the future applications for each type of lithography in order to fully understand the current techniques and their potential.

\section{Review of Current Techniques}

2.1. Hard-Mold Nanoimprint Lithography. Nanoimprint lithography has been proposed for high-throughput, high-speed patterning of nanoarrays with high resolution and low-cost. Chou et al. have suggested the concept of thermoplastic nanoimprint lithography and followed up the concept with experimental demonstration $[4,5]$. Initially, Chou et al. relied on direct mechanical deformation of the resist material under a rigid silicon mold, which exhibited resolutions beyond the limitations set by light diffractions or wavelengths. In their proof of concepts, they used silicon molds with 40 200 nm patterns and press it onto the monolayer of thermoplastic resist (poly(methyl methacrylate) (PMMA)) spin-coated on the silicon substrate (see Figure 1(a)). Furthermore, the residue of the resist was removed by reactive ion etching (RIE) process to expose the compressed areas (Figures 1(b) and 1(c)). With such technique, Chou et al. achieved maximum resolution of $\sim 25 \mathrm{~nm}$ in patterning metal dots as shown in Figure $2[4,5]$. Although silicon mold-based nanoimprint lithography approaches have proven excellent resolutions, significant challenges have risen, including difficulty in defect control of patterned area, inability to lift-off due to side wall deposition, and wearing and breakage of the mold through multiple imprint process. These limitations were thoroughly researched and improved in previous studies. We will address these three limitations and relevant improvements in following sections in detail.

2.1.1. Defect Control of Patterned Area. In this section, we are discussing one of the still remaining limitations of hard-mold nanoimprint lithography which is defect control of patterned area. Initially, only silicon substrates have been used as molds for their cost-effectiveness and simplicity to pattern master mold via electron beam lithography. However, two critical difficulties have been arisen due to wearing of master mold, and inability to conduct photo-nanoimprint lithography, which originates from opaque properties of silicon molds. In order to solve such problems, the use of working molds was proposed [53-56]. The issue of wearing of the mold because of polymer aggregation and use of working mold will be discussed further in Section 2.1.3. Furthermore, to solve the inability to conduct photo-nanoimprint lithography, the use of UV-transmittable mold was also suggested [5761]. Even though numerous kinds of materials have been used as molds, such as quartz, glass, silicon, and GeAs, for different purposes, rigid mold commonly suffers from randomized defect area where the pattern is not present. In hard-mold nanoimprint lithography, the issue with defect control is caused mainly by inflexibility of the molds, which creates the "air bubble" between the mold and the resistcoated substrates when the particles remain on the imprinted area as shown in Figure 3. Affected area by the particle ranges from few micrometers to few millimeters depending on the size of the particles [56]. Unpredictable but significant defects in the form of pinholes and thin stripes have been observed. These defects can be transferred to the final deposition and lift-off processes, which consequently results in unwanted elements such as large particles, irregular film pieces, or bare 


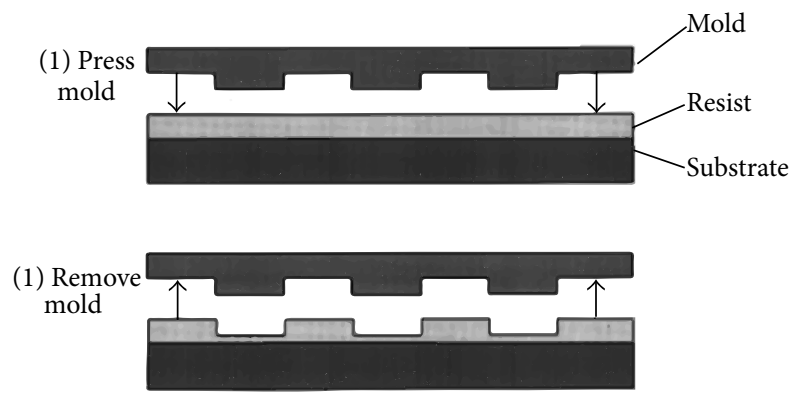

(3) RIE

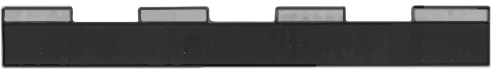

(a)

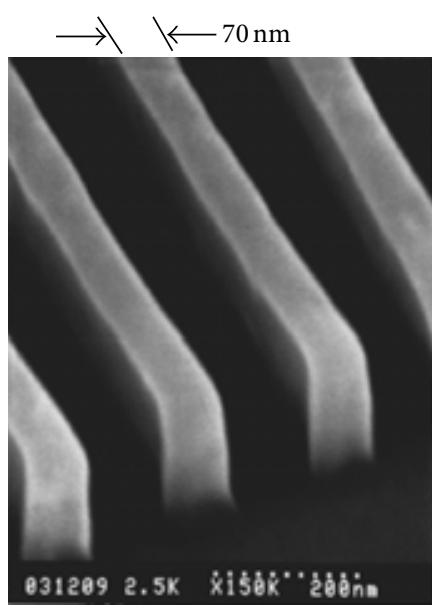

(b)

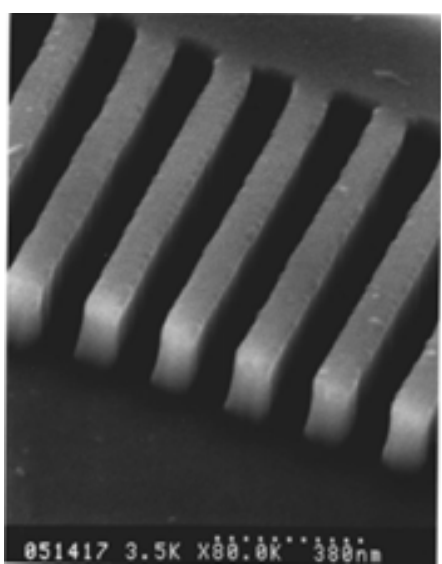

(c)

FIGURE 1: (a) Schematic of nanoimprint lithography process: (1) imprinting using a mold to create a thickness contrast in a resist, (2) mold removal, and (3) pattern transfer using anisotropic etching to remove residue resist in the compressed areas. (b) SEM micrograph of a perspective view of strips formed into a PMMA film by imprint. The strips are $70 \mathrm{~nm}$ wide and $200 \mathrm{~nm}$ tall, having a high aspect ratio, a surface roughness less than $3 \mathrm{~nm}$, and nearly perfect $90^{\circ}$ corners. (c) SEM micrograph of the mold that was used to imprint the PMMA strips shown in (b). Reproduced from [4] with permission of American Vacuum Society.

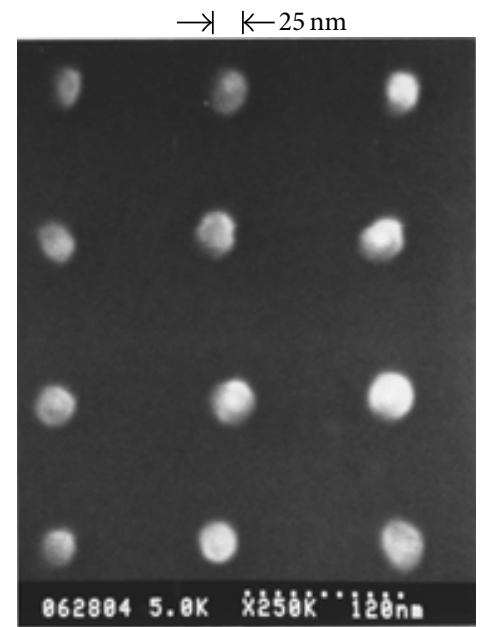

FIGURE 2: SEM micrograph of $25 \mathrm{~nm}$ diameter and $120 \mathrm{~nm}$ period metal dots fabricated by imprint lithography and a lift-off process. Reproduced from [4] with permission of American Vacuum Society.

substrate. Furthermore, this defect issue gets more significant as the mold is repeatedly used.

In order to improve the hard-mold nanoimprint lithography process, numerous efforts have been made. There have been dramatic improvements on the quality of resulting elements such as resolution, uniformity of imprint, and high imprint aspect ratio. For instance, sub-10 nm scale dot feature fabrication has been reported using a conventional hard quartz mold-based photo-nanoimprint lithography $[62,63]$. The uniformity of imprint was solved by utilizing the silicon pocket creating vacuum environment. Conventionally, the concept of the mechanical press was used in order to provide the necessary pressure to mechanically deform the resist with the mold as shown in Figure 4(a). However, since mechanical press is incapable of providing sufficient homogeneity of the forces across the whole sample area as imprinted size increases, unintended regional void between sample and mold and, further, breakage of mold due to uneven forces occur (see Figure 4(b1), (b2), (b3), and (b4)). Meanwhile, high imprint aspect ratio was also solved by using curable polymer molds such as MINS [3, 64], Ormostamp [52, 53, 56], and I-UVM series, by coating them on the hard mold, which enables replicating without any damage originating from the mold's rigidity attributed to their flexibility. These curable molds are widely used for large-area patterning with high resolution, which will be further discussed in Section 2.3.

\subsubsection{Inability to Lift-Off/Integrity Compromise due to Side} Wall Deposition. Side wall deposition has been the persistent problem during lithographic processes. Side wall deposition not only changes original topography by creating the "tail," but also hampers structure's physical properties. Furthermore, excessive side wall deposition causes complete covering of deposited metal onto the resist layer, which frustrates efficient lift-off process. Monolayer resist profile is particularly susceptible to the side wall deposition due to lack of undercut profile. Since the trajectory of the deposited atoms is random, the deposition occurs not only on the substrate, but also on the side wall of the resist patterns as shown in inset of Figure 5. As the deposition time increases, deposition completely covers the resist pattern and the substrate as one uniform coating which leads to lift-off failure.

In order to prevent such side wall deposition, two methods have been developed: (1) using highly isotropic deposition instruments and (2) preparing a double resist layer undercut profile to avoid deposition of "tail" and side wall. Firstly, current deposition equipment, such as electron 

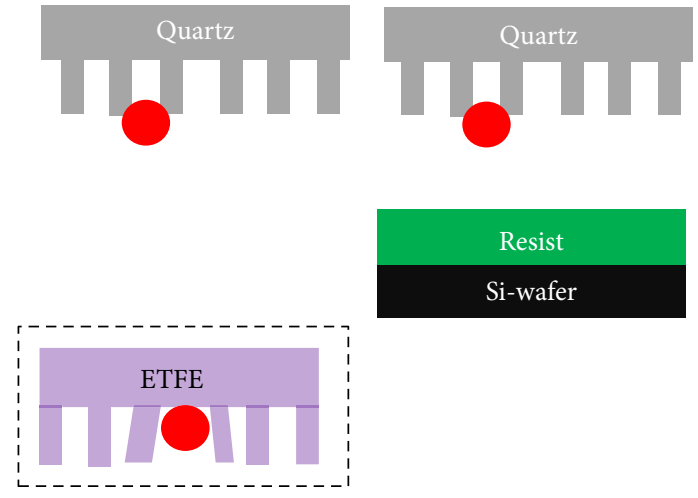

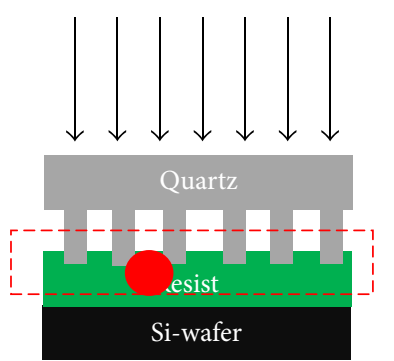

Figure 3: Schematic diagram of how a particle (red dot) can cause the air bubble between the resist and the stamp. Red dashed box shows the void area between the resist and the mold, which creates unpatterned area due to the defects; (inset) utilization of ETFE soft mold to accommodate the presence of defects.

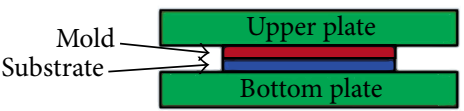

(a)

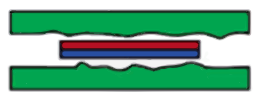

(b1)

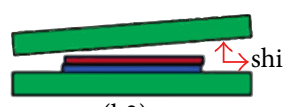

(b3)

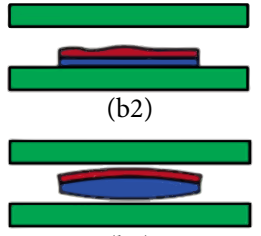

(b4)
Figure 4: (a) Schematic diagram of the solid parallel-plate press (mechanical press) nanoimprint method and potential drawbacks: (b1) failure of imprint due to imperfect plate surfaces, (b2) failure of imprint due to uneven mold/substrate backside, (b3) failure of imprint due to nonparallelism between plates, and (b4) failure of imprint due to curved sample surfaces.

beam evaporator, magnetron sputtering, ion beam sputtering, or atomic layer deposition, has different anisotropy during deposition. Figure 5 shows the typical deposition environment in the chamber of these types of equipment. As metal/oxide atoms from the source travel across the chamber, the trajectory of the deposition cannot be perfectly anisotropic. Nonzero angle vector component from the theoretical trajectory results in deposition at the side wall of the resist as shown in Figure 5. In order to solve such difficulties, following components were adjusted: (1) travel distance from the source to the sample and (2) velocity of the metal/oxide atoms travelling across the deposition chamber. As the travel distance increases, the rate of the deposition was found to be decreased with decreasing the side wall deposition as well. The velocity of the metal/oxide atoms is also critical. When the chamber is full of argon gas for deposition during sputtering process, if velocity of the metal/oxide atom is not high enough, the trajectory of the deposited atoms changes due to the collision to argon gas atoms. In order to avoid such physical phenomenon, the source power can be increased to increase velocity of atoms scattered from the target. Even though these methods can decrease the occurrence of the side wall, it still cannot completely eradicate the occurrence of the tail. As a second method, application of double resist layer undercut profile was introduced. Figure 6 shows the process of fabricating the double resist layer undercut profile. In this method, combination of RIE and wet etching to prepare an undercut profile is critical to avoid any bottom tail or undercut resist residue due to underetching [56]. Deeper undercut profile is preferred; however, too deep undercut creates the collapse of the upper resist layer as shown in Figure 7. In this regard, the combination of anisotropic deposition instrument and development of double resist layer undercut profile has been highly preferred to avoid the side wall deposition and furthermore prevent the lift-off failure. This combined one became a standard procedure for fabrication of nanosized element via nanoimprint lithography $[3,49,52,53,56]$.

2.1.3. Wearing and Breakage of the Mold through Multiple Imprint Process. After numerous optimization processes, hard-mold nanoimprint lithography has been reliably used in patterning nanosize elements in a small-area. However, persistent problem of wear and breakage of the mold through the process of imprint was not solved. Damage to the mold was primarily caused by either resist residue attached to the mold during imprint process or use of razor for demolding process. Resist residue aggregation on the surface of the mold is created by the surface hydrophilicity of the mold such as silicon, quartz, or glass. Due to this hydrophilicity of the mold, the resist favors attaching or binding to the mold surface. As a result, even after demolding, the hardened resist residue remains on the surface of the mold. The resist residue can be mostly removed with the dry $\mathrm{O}_{2}$ etching process, but certain amount of residue will still remain. This remaining resist creates defects during imprint process and regional air bubble, mentioned in Section 2.1.1. Furthermore, the amount of remaining resist increases as number of imprint processes increases, which eventually makes the mold unusable. 


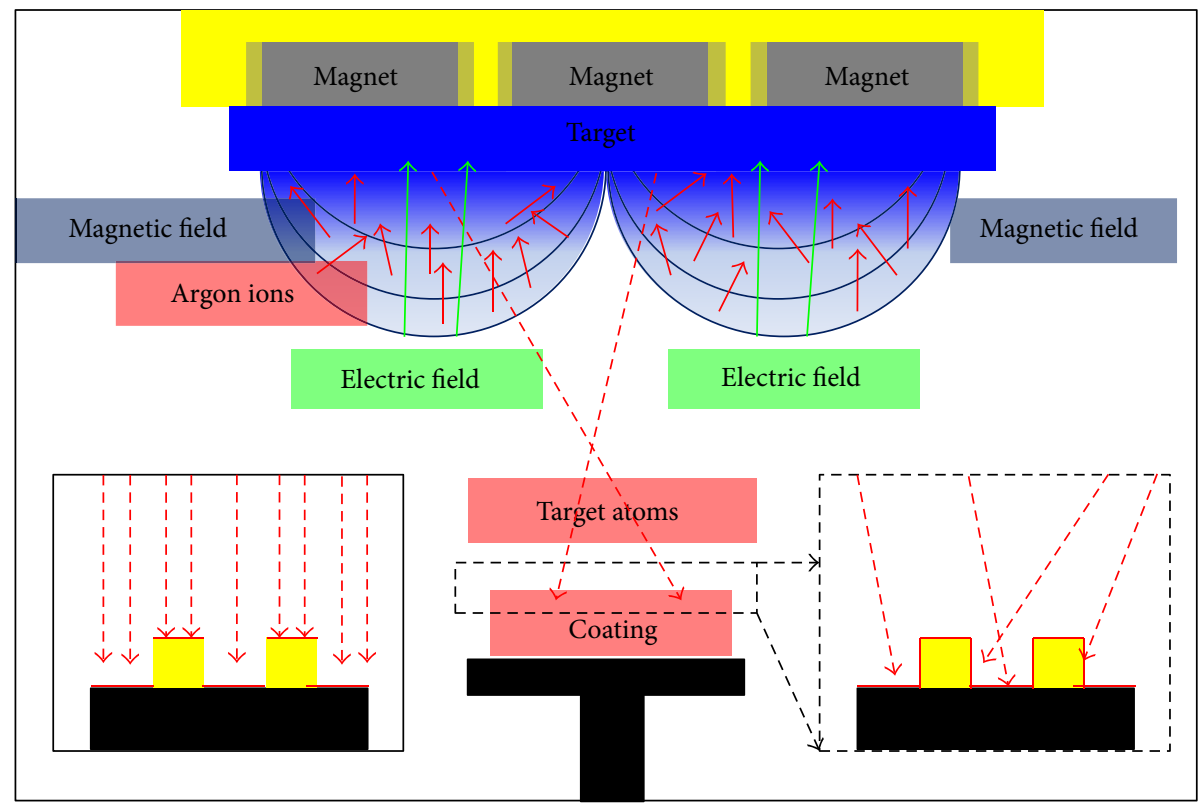

FIGURE 5: Schematic diagram of sputtering system (magnetron sputtering) showing the trajectory of the target atoms deposited on the substrate (dashed red line). As the argon ions that are bombarded on the target to cause the scattering have random velocity vector, scattered target atoms have random velocity vector. These random velocity vectors cause the side wall deposition (dashed inset). The ideal case of sputtering for optimized lift-off result is shown in solid inset.

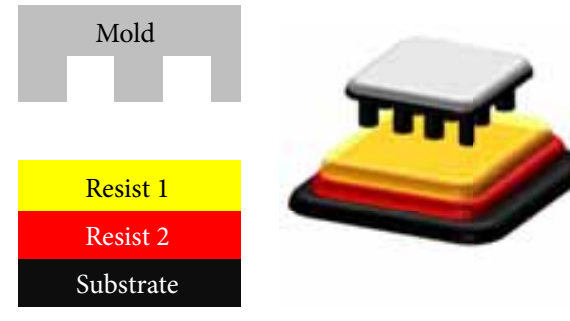

(a)

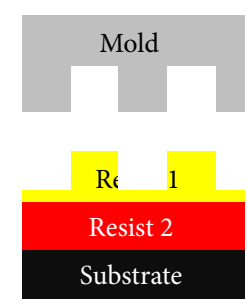

(b)

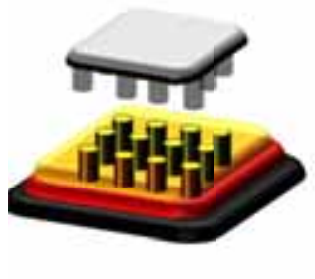

)

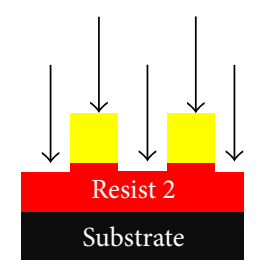

(c)

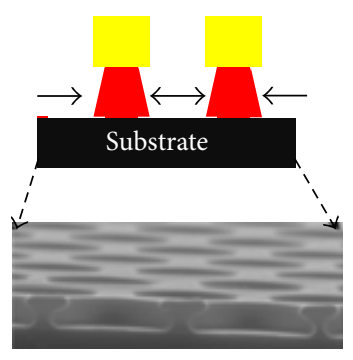

(d)

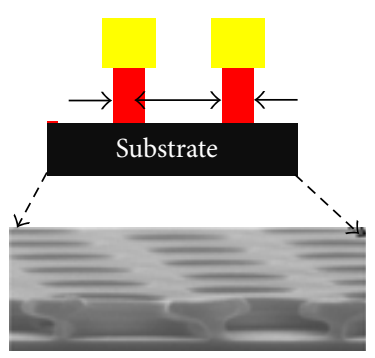

(e)

Figure 6: Process of creating a double resist layer undercut profile in order to avoid the side wall and undesired deposition. (a, b) Schematic diagram of nanoimprint. (c) showing the reactive ion etching process to expose the under resist layer; (d) and (e) showing the wet chemical etching process in order to selectively etch the under resist layer and also showing corresponding SEM images of those bilayer undercut profiles.

To solve such issues, two different methods were introduced: (1) hydrophobic coating on the surface of the hydrophilic mold to prevent the attachment of the resist to the surface (i.e., silane coating) or (2) utilization of hydrophobic polymer working mold, such as ethylene tetrafluoroethylene (ETFE), polydimethyl silane (PDMS), and polyethylene terephthalate (PET) $[3-5,52-54,56,59,62,63]$. The silane coating has been commonly used to provide very thin $(\sim<2 \mathrm{~nm})$ 


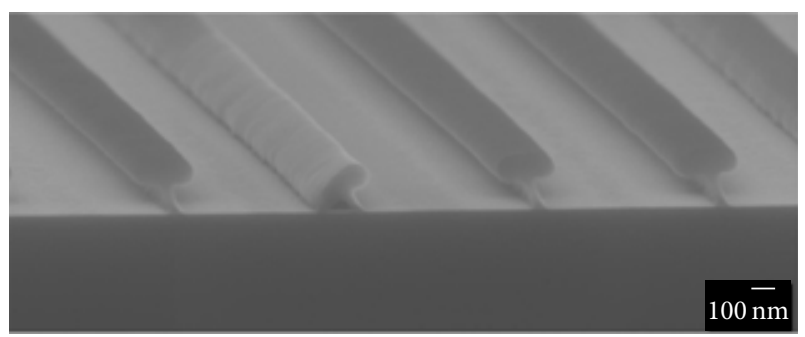

FIGURE 7: SEM image of collapsed undercut bilayer resist profile due to excessive wet etching to remove the under layer. The excess wet chemical etching can cause the under layer to be too weak to stand the weight of top layer, leading to undesirable defects.

hydrophobic-hydrophilic layer $[61,65,66]$, allowing a strong chemical bonding to surface-hydrophilic end. This chemical orientation of silane creates a strong chemical monolayer self-assembly on the surface $[61,65,66]$ and endows high hydrophobicity to the surface. The process of silane coating is mostly based on the evaporation process in desiccators and let the evaporated miniscules coated on $[61,65,66]$. Currently hydrophobic coating on the silicon, glass, or quartz molds has been the standard process for minimizing defects and damage caused on both the patterned surface and the mold $[3,52,53,56,61,65,66]$. On the other hand, sudden breakage has been another problem during the process of demolding. After mold is pressed onto the substrate with high pressure, it is very difficult to separate the mold from the substrate clearly because of the rigidity of both the mold and substrate. Conventionally, razor blade has been applied to separate the mold from the substrate. However, razor blade often introduces the surface damage to the mold or substrate in the process of demolding, which makes the mold and substrate very susceptible to crack in the further processes. This has been observed frequently during the imprint process during the hard mold $[52,53,56]$. In order to avoid such issues, flexible mold was introduced. Instead of hard glass, quartz, or silicon molds, ETFE, PDMS, or PET-based soft molds were used for compensating hard mold's rigidity and preventing the risk of both mold's and substrate's breakage during demolding process.

2.2. Soft-Mold Nanoimprint Lithography. Soft-mold nanoimprint lithography has been developed to increase the yield and defect control in patterning. As mentioned, despite high resolution, hard-mold nanoimprint lithography has numerous problems that are associated with the inherent properties of hard mold. The properties of patterned elements are easily influenced by extrinsic parameters, such as defects generated from the lithography process. For instance, submicron-size particles in the resist solution that are not filtered out can be transferred onto the substrate after spin-coating, causing the failure of lithography. The consequence of such defects has introduced significant issues for nanoimprint lithography, in particular, during mechanical contact between a stamp and a resist for pattern generation. As a result, as mentioned in Section 2.1.3 the mold can be easily damaged. These issues introduce a profound dilemma: a mold should be used as much as possible for pattern generation, maintaining the integrity of the pattern, but the mold should not be considered as consumable for its high-cost. Replication of the hard mold can be made using soft and flexible material, often polymer-based materials, such as PDMS, PFPE, ETFE, and PET, to mediate this dilemma. In addition to their low-cost, their flexibility provides conformal and intimate contact between substrate and mold without the need for high external pressure. Also, their flexibility also makes them insensitive to particle contaminants in the resist, as they can locally deform around a particle without damaging the mold or affecting the lithography yield [51,67-73]. Now, we will focus on two popular materials for soft mold, PDMS and ETFE.

2.2.1. PDMS. PDMS $\left(\mathrm{CH}_{3}\left[\mathrm{Si}\left(\mathrm{CH}_{3}\right)_{2} \mathrm{O}\right]_{n} \mathrm{Si}\left(\mathrm{CH}_{3}\right)_{3}\right)$ is one of the earliest and most widely used polymers for soft-mold material. There are certain reasons that PDMS emerged as a standard material for the soft mold. That is, PDMS has a low Young's modulus ( 2.0 Mpa) and low surface energy $\left(\sim 16 \mathrm{~mJ} / \mathrm{m}^{2}\right)$ that allows for conformal contact and easy release from both a master mold and imprinted patterns. Furthermore, PDMS demonstrates relatively high toughness with a high elongation at break $(>150 \%)$ which provides great degree of flexibility during patterning conditions [53, $56,74,75]$. Furthermore, it has high gas permeability, which allows the air trapped between the soft mold and the imprinted substrate to be released through the soft mold itself. This is one of the significant advantages of PDMS which enables effective large-area patterning. However, low Young's modulus of PDMS often limits the replication of both the high-density and high resolution features and, therefore, is detrimental for forming high aspect ratio structures as fabricating such features will be very susceptible to collapse, deformation, or merge. In addition, PDMS is a very porous material so that organic solvents or monomers easily penetrate into it. This causes regional surface inhomogeneity in the resist composition and consequently leads to swelling of the mold. This will be a critical problem when trying to pattern biological materials or functionalized materials with controlled surface. Poor solvent resistance also has a serious effect on reproducibility due to degradation in the course of repeated patterning. These difficulties, despite of the flexibility and conformality, limit the application of PDMS as an ultimate form of the mold for further applications.

2.2.2. ETFE. PDMS has demonstrated the utilization and potentials of soft molds; however it also has shown the critical limitations for repeatable high resolution imprint, caused by high porosity, low Young's modulus, and low reproducibility. Regarding these issues, many efforts have been made in developing molds maintaining flexibility and conformality, with securing high Young's modulus, and high durability against thermal and chemical environment. For instance, ETFE $\left(\mathrm{C}_{4} \mathrm{H}_{4} \mathrm{~F}_{4}\right)_{n}$ is a recently found fluorinated polymer material which has been used for soft molds for its high Young modulus (>10 Mpa), good mechanical strength, good 

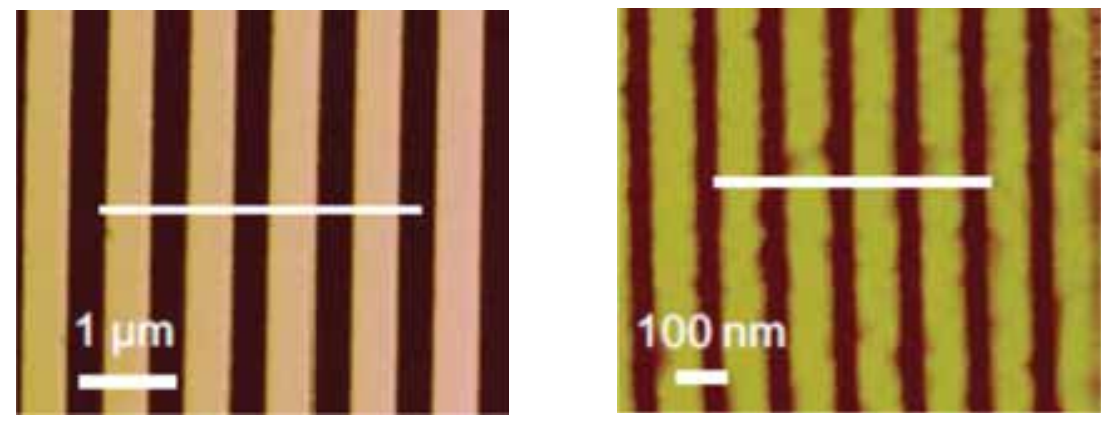

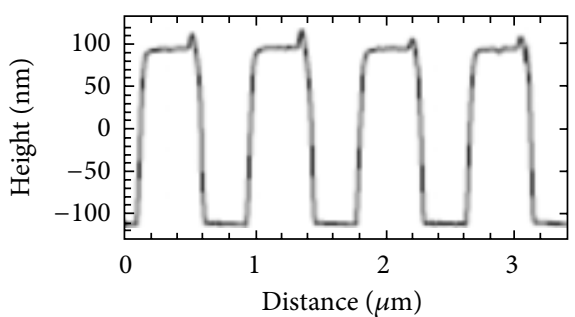

(a)

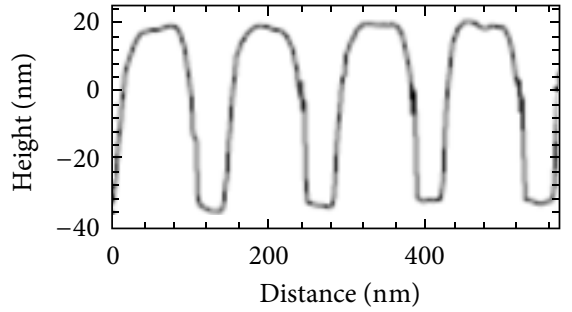

(b)
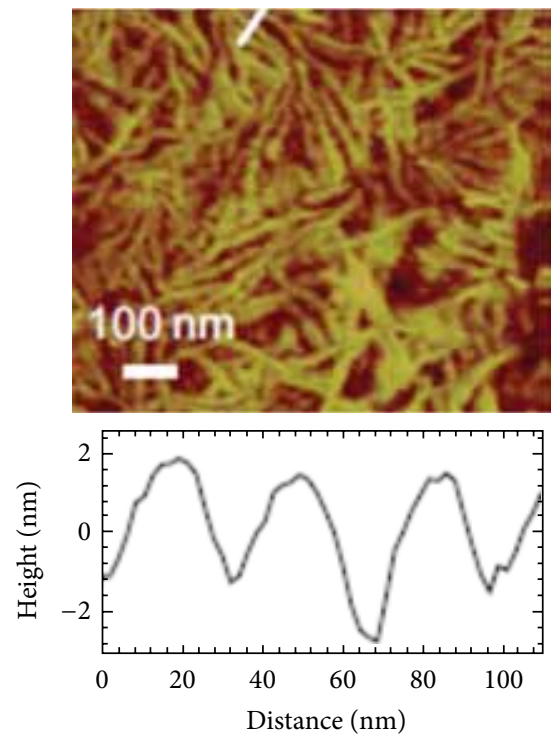

(c)

Figure 8: AFM topography images and profiles (along white lines) of ETFE molds with (a) $833 \mathrm{~nm}$ period and (b) $139 \mathrm{~nm}$ period and (c) nominally flat mold showing needlelike crystals. Reproduced from [49] with permission of American Vacuum Society.

chemical stability, and very low surface energy. ETFE was firstly used to replace PDMS-based polymers for realizing cleaner and finer soft-mold nanoimprint lithography [49, $56,69,73]$. Furthermore, Barbero et al. demonstrated ETFE stamps for high-temperature nanoimprint lithography [69]. Despite these advantages compared to PDMS or Teflon such as exceptional toughness and flexibility, high thermal stability, and superior mechanical properties $[49,56,69$, 73], patterning resolution has been limited because of the crystallization of the polymer, which happens at $\sim 100 \mathrm{~nm}$ scale. This crystallization has been observed in previous study [49], showing the crystalline domains spaced $20 \sim 40 \mathrm{~nm}$ apart, with a height between $2.5 \mathrm{~nm}$ and $5 \mathrm{~nm}$ as shown in
Figure 8 . These crystalline grains are likely accountable for losing integrity of imprinted feature with $<150 \mathrm{~nm}$ resolution.

To further improve the fabrication resolution but at the same time retain all the advantages that these soft molds have demonstrated, hybrid mold, which combines the UV-curable hard mold and soft mold, has been introduced.

2.3. UV-Curable Hybrid-Mold Nanoimprint Lithography. UV-curable hybrid mold is composed of transparent flexible substrate, often PET, and UV-curable resin, which can be coated on the flexible substrate and work as a patterned mold after curing. This mechanism was initially introduced for the purpose of increasing mold's patterning resolution 

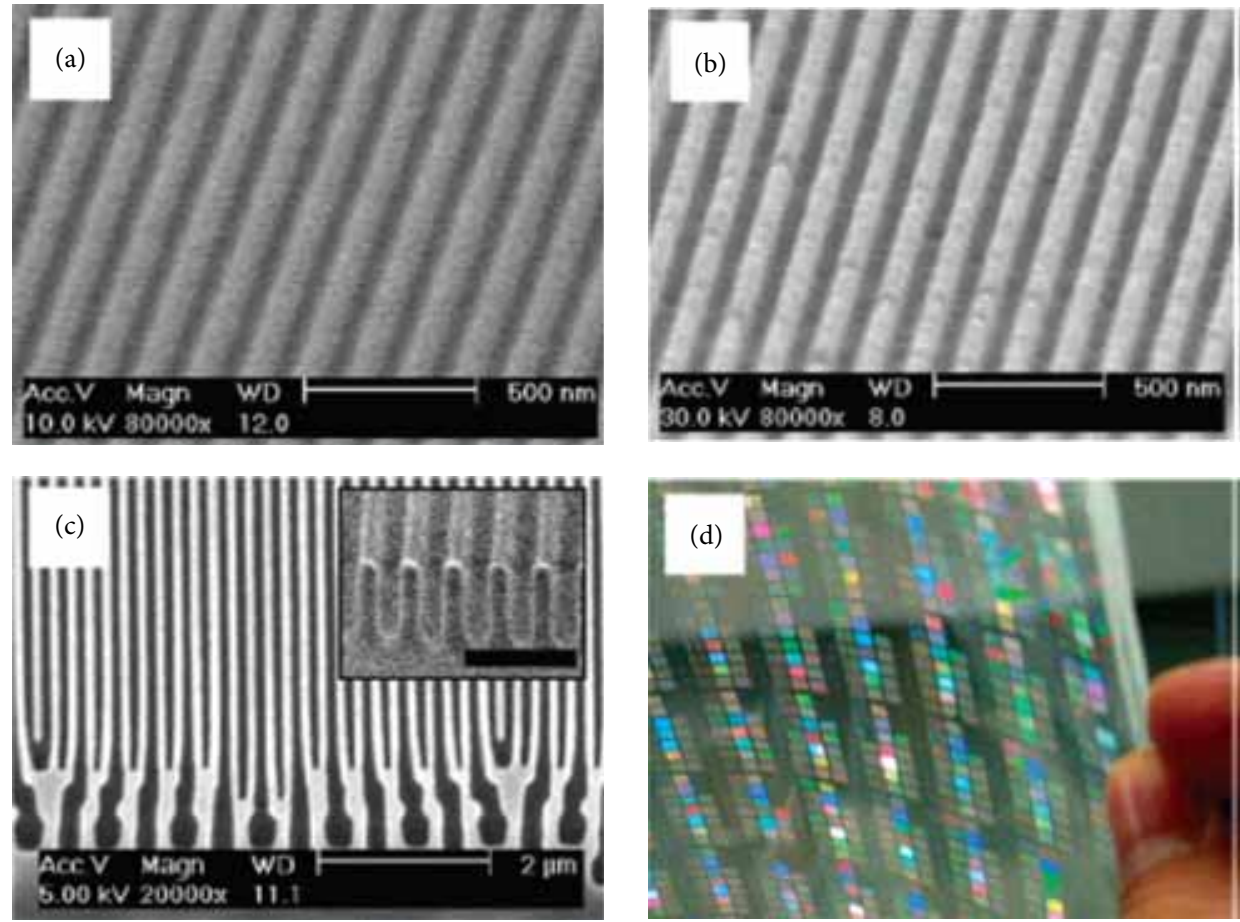

FIGURE 9: SEM and optical microscopic images of replication results. (a) Master pattern of $75 \mathrm{~nm}$ line space polarizer. (b) Replicated pattern of (a). (c) Replicated pattern of a $100 \mathrm{~nm}$ line space circuit pattern. Inset is the cross-sectional SEM images. Bar scale in the inset is $500 \mathrm{~nm}$. (d) Example of large-area replication of hologram gratings. Reproduced from permission from [3]. Copyright 2004 American Chemical Society.

which has been heavily limited by the polymer crystallization. Therefore, UV-curable mold nanoimprint lithography has been introduced as one of spin-off to solve the issues arising from the conventional soft-mold nanoimprint lithography $[3,52,53,56,64]$. Firstly, conventional soft mold only uses deformable polymer as a mold for patterning, and therefore, the resolution and surface chemistry totally depended upon the chemical structure of the polymer used for the mold. However, UV-curable mold completely resolves this problem by taking advantage of its high young modulus and great mechanical strength after curing, and it can be very thinly coated on the surface of flexible mold that it also retains the flexibility of the soft mold. For instance, Choi et al. have introduced the UV-curable mold, so called MINS, which consists of a functionalized prepolymer with acrylate group, a photoinitiator, and a radiation-curable releasing agent for the surface activity. This material has a number of desirable properties such as mechanical rigidity ( $>40 \mathrm{Mpa})$, flexibility, small shrinkage, and light transmittance to UV [3]. This material, after cured, has shown $>40 \mathrm{Mpa}$ tensile modulus (cf. 1.8 MPa for PDMS or 8.2 MPa for hard PDMS), and the elongation at break is $31 \%$ [3]. With these desirable qualities, they have demonstrated sub-100 nm structures effectively, as shown in Figure 9. Other hybrid molds, such as Ormostamp and I-UVM-100, also have demonstrated very similar desirable traits with sub-100 $\mathrm{nm}$ and furthermore, in some cases, sub-50 nm fabrication in large area. Furthermore, the hybrid molds have been utilized for the largearea patterning, such as low pressure roll-to-roll imprint application. Since hybrid molds provide the great degree of flexibility, low-cost fabrication, and its durability, low pressure roll-to-roll nanoimprint lithographic technique has been suggested in order to pattern very large area $(>1 \mathrm{ft} \times$ $1 \mathrm{ft}$ scale) $[48,55,76]$. The previous study has demonstrated that, with low pressure from simple roll-to-roll instrument $(<200 \mathrm{~N})$, the nanoscale patterns $(<100 \mathrm{~nm})$ can be fabricated $[48,55,76]$. This provides the potential for industry nanoscale fabrication method using hybrid mold since this particular mold provides high resolution, large scale patterning, lowcost, and easiness for patterning. As the optimization and improvement on these molds continue, we are expecting the nanoimprint resolution to be higher and imprint area to be larger significantly.

\section{Conclusion and Perspectives}

In this review, we summarized the status of current technology for mold fabrication used in nanoimprint lithography (Table 1). Although conventional lithographic techniques such as electron beam lithography, photolithography, and atomic force microscopy (AFM) lithography are good choices for single-nanostructure devices with resolution down to tens of nanometers their limitation of extensive writing and patterning times critically limits the application for high volume production. In particular, photolithography has been proposed to be compatible with large-area and fast patterning. However, the resolution limits also hinder this method to be prevalently used as the popular means for fabricating 


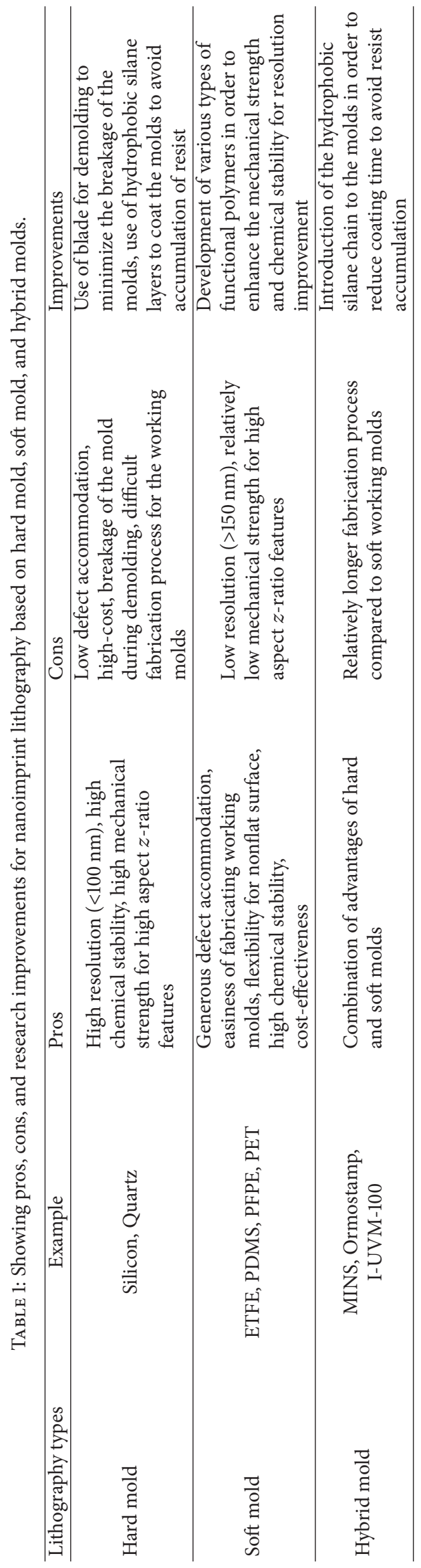


nanometer scale devices. In this regard, nanoimprint lithography has demonstrated the potential to achieve both high volume production and very high resolution. Conventional hard-mold lithography has shown very high resolution in tens of nanometers and thus, sub-100 nm magnetic devices (i.e., AMR, TMR, or GMR devices) or sub-100 nm electronic devices (i.e., memristor or selector devices) have been fabricated using hard molds. However, randomized defects due to the presence of the particle in atmosphere and easy wear and degradation of the hard mold have significantly limited their potential applications. Even though soft mold has solved these problems of hard mold, the chemical structure of the polymer used for soft mold still limited the potential to be used for high resolution lithography. Therefore, soft mold has been often utilized in biomedical applications such as preparation of biocompatible agents (i.e., iron oxide nanoparticles) since soft mold can effectively generate sub$500 \mathrm{~nm}$ uniform nanoparticles in mass production. Hybrid mold using UV-curable resin has been suggested to achieve very high resolution ( $<10 \mathrm{~nm}$ fabrication) but at the same time retain all the great advantages of soft mold such as flexibility and conformality. They are still being developed to push the resolution limit down to several nanometers and further subnanometers. They are expected to come across as a novel nanoscale fabrication method, improving the capability of the lithographic technique for the advancement of the technology.

\section{Competing Interests}

The authors declare that they have no competing interests.

\section{Acknowledgments}

This research was supported by Basic Science Research Program through the National Research Foundation (NRF) funded by the Ministry of Science, ICT \& Future Planning (2015R1C1A1A01053241).

\section{References}

[1] D. A. Canelas, K. P. Herlihy, and J. M. DeSimone, "Top-down particle fabrication: control of size and shape for diagnostic imaging and drug delivery," Wiley Interdisciplinary Reviews: Nanomedicine and Nanobiotechnology, vol. 1, no. 4, pp. 391-404, 2009.

[2] R. Chen, T.-T. D. Tran, K. W. Ng et al., "Nanolasers grown on silicon," Nature Photonics, vol. 5, no. 3, pp. 170-175, 2011.

[3] S.-J. Choi, P. J. Yoo, S. J. Baek, T. W. Kim, and H. H. Lee, "An ultraviolet-curable mold for Sub-100-nm lithography," Journal of the American Chemical Society, vol. 126, no. 25, pp. 77447745, 2004.

[4] S. Y. Chou, P. R. Krauss, and P. J. Renstrom, "Nanoimprint lithography," Journal of Vacuum Science and Technology B: Microelectronics and Nanometer Structures, vol. 14, no. 6, pp. 4129-4133, 1996.

[5] S. Y. Chou, P. R. Krauss, and P. J. Renstrom, "Imprint lithography with 25-nanometer resolution," Science, vol. 272, no. 5258, pp. 85-87, 1996.
[6] R. Ferris, A. Hucknall, B. S. Kwon, T. Chen, A. Chilkoti, and S. Zauscher, "Field-induced nanolithography for patterning of non-fouling polymer brush surfaces," Small, vol. 7, no. 21, pp. 3032-3037, 2011.

[7] R. Garcia, R. V. Martinez, and J. Martinez, "Nano-chemistry and scanning probe nanolithographies," Chemical Society Reviews, vol. 35, no. 1, pp. 29-38, 2006.

[8] W. Hu, R. J. Wilson, C. M. Earhart, A. L. Koh, R. Sinclair, and S. X. Wang, "Synthetic antiferromagnetic nanoparticles with tunable susceptibilities," Journal of Applied Physics, vol. 105, no. 7, Article ID 07B508, 2009.

[9] W. Hu, R. J. Wilson, A. Koh et al., "High-moment antiferromagnetic nanoparticles with tunable magnetic properties," Advanced Materials, vol. 20, no. 8, pp. 1479-1483, 2008.

[10] W. Hu, M. Zhang, R. J. Wilson et al., "Fabrication of planar, layered nanoparticles using tri-layer resist templates," Nanotechnology, vol. 22, no. 18, Article ID 185302, 2011.

[11] D.-H. Kim, E. A. Rozhkova, I. V. Ulasov et al., "Biofunctionalized magnetic-vortex microdiscs for targeted cancer-cell destruction," Nature Materials, vol. 9, no. 2, pp. 165-171, 2010.

[12] H. Ko, K. Takei, R. Kapadia et al., "Ultrathin compound semiconductor on insulator layers for high-performance nanoscale transistors," Nature, vol. 468, no. 7321, pp. 286-289, 2010.

[13] J.-H. Lee, Y.-M. Huh, Y.-W. Jun et al., "Artificially engineered magnetic nanoparticles for ultra-sensitive molecular imaging," Nature Medicine, vol. 13, no. 1, pp. 95-99, 2007.

[14] E. A. Vitol, V. Novosad, and E. A. Rozhkova, "Multifunctional ferromagnetic disks for modulating cell function," IEEE Transactions on Magnetics, vol. 48, no. 11, pp. 3269-3274, 2012.

[15] J. Yoon, S. Jo, I. S. Chun et al., "GaAs photovoltaics and optoelectronics using releasable multilayer epitaxial assemblies," Nature, vol. 465, no. 7296, pp. 329-333, 2010.

[16] T. Hyeon, S. S. Lee, J. Park, Y. Chung, and H. B. Na, "Synthesis of highly crystalline and monodisperse maghemite nanocrystallites without a size-selection process," Journal of the American Chemical Society, vol. 123, no. 51, pp. 12798-12801, 2001.

[17] M. M. Lin, H.-H. Kim, M. Muhammed, and D. K. Kim, "Iron oxide-based nanomagnets in nanomedicine: fabrication and applications," Nano Reviews, vol. 1, article 4883, 2010.

[18] Z. L. Liu, Y. J. Liu, K. L. Yao, Z. H. Ding, J. Tao, and X. Wang, "Synthesis and magnetic properties of $\mathrm{Fe}_{3} \mathrm{O}_{4}$ nanoparticles," Journal of Materials Synthesis and Processing, vol. 10, no. 2, pp. 83-87, 2002.

[19] R. Massart, "Preparation of aqueous magnetic liquids in alkaline and acidic media," IEEE Transactions on Magnetics, vol. 17, no. 2, pp. 1247-1248, 1981.

[20] C. Xu, X. Wang, and J. Zhu, "Graphene-metal particle nanocomposites," Journal of Physical Chemistry C, vol. 112, no. 50, pp. 19841-19845, 2008.

[21] M. T. Reetz and W. Helbig, "Size-selective synthesis of nanostructured transition metal clusters," Journal of the American Chemical Society, vol. 116, pp. 7401-7402, 1994.

[22] Y.-Y. Yu, S.-S. Chang, C.-L. Lee, and C. R. C. Wang, "Gold nanorods: electrochemical synthesis and optical properties," The Journal of Physical Chemistry B, vol. 101, no. 34, pp. 66616664, 1997.

[23] V. Subramanian, E. E. Wolf, and P. V. Kamat, "Catalysis with $\mathrm{TiO}_{2}$ /gold nanocomposites. Effect of metal particle size on the fermi level equilibration," Journal of the American Chemical Society, vol. 126, no. 15, pp. 4943-4950, 2004. 
[24] A. K. Gupta and M. Gupta, "Synthesis and surface engineering of iron oxide nanoparticles for biomedical applications," Biomaterials, vol. 26, no. 18, pp. 3995-4021, 2005.

[25] M. Hirata, T. Gotou, S. Horiuchi, M. Fujiwara, and M. Ohba, "Thin-film particles of graphite oxide 1: high-yield synthesis and flexibility of the particles," Carbon, vol. 42, no. 14, pp. 2929-2937, 2004.

[26] M. K. Kennedy, F. E. Kruis, H. Fissan, B. R. Mehta, S. Stappert, and G. Dumpich, "Tailored nanoparticle films from monosized tin oxide nanocrystals: particle synthesis, film formation, and size-dependent gas-sensing properties," Journal of Applied Physics, vol. 93, no. 1, pp. 551-560, 2003.

[27] G. Moad, E. Rizzardo, and S. H. Thang, "Radical additionfragmentation chemistry in polymer synthesis," Polymer, vol. 49, no. 5, pp. 1079-1131, 2008.

[28] C. J. Hawker, A. W. Bosman, and E. Harth, "New polymer synthesis by nitroxide mediated living radical polymerizations," Chemical Reviews, vol. 101, no. 12, pp. 3661-3688, 2001.

[29] R. H. Grubbs and W. Tumas, "Polymer synthesis and organotransition metal chemistry," Science, vol. 243, no. 4893, pp. 907-915, 1989.

[30] A. I. Cooper, "Polymer synthesis and processing using supercritical carbon dioxide," Journal of Materials Chemistry, vol. 10, no. 2, pp. 207-234, 2000.

[31] C. E. Hoyle, A. B. Lowe, and C. N. Bowman, "Thiol-click chemistry: a multifaceted toolbox for small molecule and polymer synthesis," Chemical Society Reviews, vol. 39, no. 4, pp. 1355-1387, 2010.

[32] D. Byrom, "Polymer synthesis by microorganisms: technology and economics," Trends in Biotechnology, vol. 5, no. 9, pp. 246250, 1987.

[33] G. M. Whitesides, J. P. Mathias, and C. T. Seto, "Molecular self-assembly and nanochemistry: a chemical strategy for the synthesis of nanostructures," DTIC Document, 1991.

[34] O. Prucker and J. Rühe, "Synthesis of poly(styrene) monolayers attached to high surface area silica gels through self-assembled monolayers of azo initiators," Macromolecules, vol. 31, no. 3, pp. 592-601, 1998.

[35] T. R. Cook, Y.-R. Zheng, and P. J. Stang, "Metal-organic frameworks and self-assembled supramolecular coordination complexes: comparing and contrasting the design, synthesis, and functionality of metal-organic materials," Chemical Reviews, vol. 113, no. 1, pp. 734-777, 2013.

[36] Y. Zhou and M. Antonietti, "Synthesis of very small $\mathrm{TiO}_{2}$ nanocrystals in a room-temperature ionic liquid and their selfassembly toward mesoporous spherical aggregates," Journal of the American Chemical Society, vol. 125, no. 49, pp.14960-14961, 2003.

[37] K.-Q. Peng, Y.-J. Yan, S.-P. Gao, and J. Zhu, "Synthesis of largearea silicon nanowire arrays via self-assembling nanoelectrochemistry," Advanced Materials, vol. 14, no. 16, pp. 1164-1167, 2002.

[38] I. Martínez-Mera, M. E. Espinosa-Pesqueira, R. PérezHernández, and J. Arenas-Alatorre, "Synthesis of magnetite $\left(\mathrm{Fe}_{3} \mathrm{O}_{4}\right)$ nanoparticles without surfactants at room temperature," Materials Letters, vol. 61, no. 23-24, pp. 44474451, 2007.

[39] K. V. P. M. Shafi, A. Ulman, X. Yan et al., "Sonochemical synthesis of functionalized amorphous iron oxide nanoparticles," Langmuir, vol. 17, no. 16, pp. 5093-5097, 2001.

[40] R. F. Pease, "Electron beam lithography," Contemporary Physics, vol. 22, no. 3, pp. 265-290, 1981.
[41] C. Vieu, F. Carcenac, A. Pépin et al., "Electron beam lithography: resolution limits and applications," Applied Surface Science, vol. 164, no. 1-4, pp. 111-117, 2000.

[42] A. A. Tseng, K. Chen, C. D. Chen, and K. J. Ma, "Electron beam lithography in nanoscale fabrication: recent development," IEEE Transactions on Electronics Packaging Manufacturing, vol. 26, no. 2, pp. 141-149, 2003.

[43] A. N. Broers, A. C. F. Hoole, and J. M. Ryan, "Electron beam lithography-resolution limits," Microelectronic Engineering, vol. 32, no. 1-4, pp. 131-142, 1996.

[44] W. Chen and H. Ahmed, "Fabrication of 5-7 nm wide etched lines in silicon using $100 \mathrm{keV}$ electron-beam lithography and polymethylmethacrylate resist," Applied Physics Letters, vol. 62, no. 13, pp. 1499-1501, 1993.

[45] K. Liu, P. Avouris, J. Bucchignano, R. Martel, S. Sun, and J. Michl, "Simple fabrication scheme for sub-10 nm electrode gaps using electron-beam lithography," Applied Physics Letters, vol. 80, no. 5, pp. 865-867, 2002.

[46] T. A. Lowrey and R. W. Chance, "Reduced mask manufacture of semiconductor memory devices," Goggle Patents, 1991.

[47] E. Yablonovitch and R. B. Vrijen, "Optical projection lithography at half the Rayleigh resolution limit by two-photon exposure," Optical Engineering, vol. 38, no. 2, pp. 334-338, 1999.

[48] S. H. Ahn and L. J. Guo, "High-speed roll-to-roll nanoimprint lithography on flexible plastic substrates," Advanced Materials, vol. 20, no. 11, pp. 2044-2049, 2008.

[49] D. N. Weiss, S. T. Meyers, and D. A. Keszler, "All-inorganic thermal nanoimprint process," Journal of Vacuum Science \& Technology B: Nanotechnology and Microelectronics, vol. 28, no. 4, pp. 823-828, 2010.

[50] N. Koo, U. Plachetka, M. Otto et al., "The fabrication of a flexible mold for high resolution soft ultraviolet nanoimprint lithography," Nanotechnology, vol. 19, no. 22, Article ID 225304, 2008.

[51] N. Koo, M. Bender, U. Plachetka et al., "Improved mold fabrication for the definition of high quality nanopatterns by soft UV-nanoimprint lithography using diluted PDMS material," Microelectronic Engineering, vol. 84, no. 5-8, pp. 904-908, 2007.

[52] L. J. Guo, "Recent progress in nanoimprint technology and its applications," Journal of Physics D: Applied Physics, vol. 37, no. 11, pp. R123-R141, 2004.

[53] L. J. Guo, "Nanoimprint lithography: methods and material requirements," Advanced Materials, vol. 19, no. 4, pp. 495-513, 2007.

[54] A. Lebib, Y. Chen, J. Bourneix et al., "Nanoimprint lithography for a large area pattern replication," Microelectronic Engineering, vol. 46, no. 1, pp. 319-322, 1999.

[55] S. H. Ahn and L. J. Guo, "Large-area roll-to-roll and roll-toplate Nanoimprint Lithography: a step toward high-throughput application of continuous nanoimprinting," ACS Nano, vol. 3, no. 8, pp. 2304-2310, 2009.

[56] W. Zhang and K. M. Krishnan, "Epitaxial patterning of thin-films: conventional lithographies and beyond," Journal of Micromechanics and Microengineering, vol. 24, no. 9, Article ID 093001, 2014.

[57] M. Bender, M. Otto, B. Hadam, B. Spangenberg, and H. Kurz, "Multiple imprinting in UV-based nanoimprint lithography: related material issues," Microelectronic Engineering, vol. 61-62, pp. 407-413, 2002.

[58] Q. Xia, C. Keimel, H. Ge, Z. Yu, W. Wu, and S. Y. Chou, "Ultrafast patterning of nanostructures in polymers using laser 
assisted nanoimprint lithography," Applied Physics Letters, vol. 83, no. 21, pp. 4417-4419, 2003.

[59] B. Vratzov, A. Fuchs, M. Lemme, W. Henschel, and H. Kurz, "Large scale ultraviolet-based nanoimprint lithography," Journal of Vacuum Science \& Technology B, vol. 21, no. 6, pp. 27602764, 2003.

[60] M. Bender, M. Otto, B. Hadam, B. Vratzov, B. Spangenberg, and H. Kurz, "Fabrication of nanostructures using a UV-based imprint technique," Microelectronic Engineering, vol. 53, no. 1, pp. 233-236, 2000.

[61] H. Schift, "Nanoimprint lithography: an old story in modern times? A review," Journal of Vacuum Science and Technology B: Microelectronics and Nanometer Structures, vol. 26, no. 2, pp. 458-480, 2008.

[62] W. Wu, W. M. Tong, J. Bartman et al., "Sub-10 nm nanoimprint lithography by wafer bowing," Nano Letters, vol. 8, no. 11, pp. 3865-3869, 2008.

[63] S. Y. Chou, P. R. Krauss, W. Zhang, L. Guo, and L. Zhuang, "Sub$10 \mathrm{~nm}$ imprint lithography and applications," Journal of Vacuum Science and Technology B: Microelectronics and Nanometer Structures, vol. 15, no. 6, pp. 2897-2904, 1997.

[64] B. K. Lee, L.-Y. Hong, H. Y. Lee, D.-P. Kim, and T. Kawai, "Replica mold for nanoimprint lithography from a novel hybrid resin," Langmuir, vol. 25, no. 19, pp. 11768-11776, 2009.

[65] H. Schift, S. Saxer, S. Park, C. Padeste, U. Pieles, and J. Gobrecht, "Controlled co-evaporation of silanes for nanoimprint stamps," Nanotechnology, vol. 16, no. 5, pp. S171-S175, 2005.

[66] S. Park, H. Schift, C. Padeste, B. Schnyder, R. Ü. Kötz, and J. Gobrecht, "Anti-adhesive layers on nickel stamps for nanoimprint lithography," Microelectronic Engineering, vol. 73-74, pp. 196-201, 2004.

[67] S. H. Ko, I. Park, H. Pan et al., "Direct nanoimprinting of metal nanoparticles for nanoscale electronics fabrication," Nano Letters, vol. 7, no. 7, pp. 1869-1877, 2007.

[68] M. G. Kang, M. S. Kim, J. Kim, and L. J. Guo, "Organic solar cells using nanoimprinted transparent metal electrodes," Advanced Materials, vol. 20, pp. 4408-4413, 2008.

[69] D. R. Barbero, M. S. M. Saifullah, P. Hoffmann et al., "High resolution nanoimprinting with a robust and reusable polymer mold," Advanced Functional Materials, vol. 17, no. 14, pp. 24192425, 2007.

[70] S. H. Ahn, J.-S. Kim, and L. J. Guo, "Bilayer metal wire-grid polarizer fabricated by roll-to-roll nanoimprint lithography on flexible plastic substrate," Journal of Vacuum Science \& Technology B, vol. 25, no. 6, pp. 2388-2391, 2007.

[71] J. John, Y. Tang, J. P. Rothstein, J. J. Watkins, and K. R. Carter, "Large-area, continuous roll-to-roll nanoimprinting with PFPE composite molds," Nanotechnology, vol. 24, no. 50, Article ID 505307, 2013.

[72] S. Gilles, M. Meier, M. Prömpers et al., "UV nanoimprint lithography with rigid polymer molds," Microelectronic Engineering, vol. 86, no. 4-6, pp. 661-664, 2009.

[73] D. N. Weiss, H.-C. Yuan, B. G. Lee et al., "Nanoimprinting for diffractive light trapping in solar cells," Journal of Vacuum Science \& Technology B, vol. 28, Article ID C6M98, 2010.

[74] J. K. Kim, H. S. Cho, H.-S. Jung et al., "Effect of surface tension and coefficient of thermal expansion in $30 \mathrm{~nm}$ scale nanoimprinting with two flexible polymer molds," Nanotechnology, vol. 23, no. 23, Article ID 235303, 2012.

[75] A. L. Thangawng, M. A. Swartz, M. R. Glucksberg, and R. S. Ruoff, "Bond-detach lithography: a method for micro/nanolithography by precision PDMS patterning," Small, vol. 3, no. 1, pp. 132-138, 2007.

[76] S. Ahn, J. Cha, H. Myung, S.-M. Kim, and S. Kang, "Continuous ultraviolet roll nanoimprinting process for replicating largescale nano- and micropatterns," Applied Physics Letters, vol. 89, no. 21, Article ID 213101, 2006. 

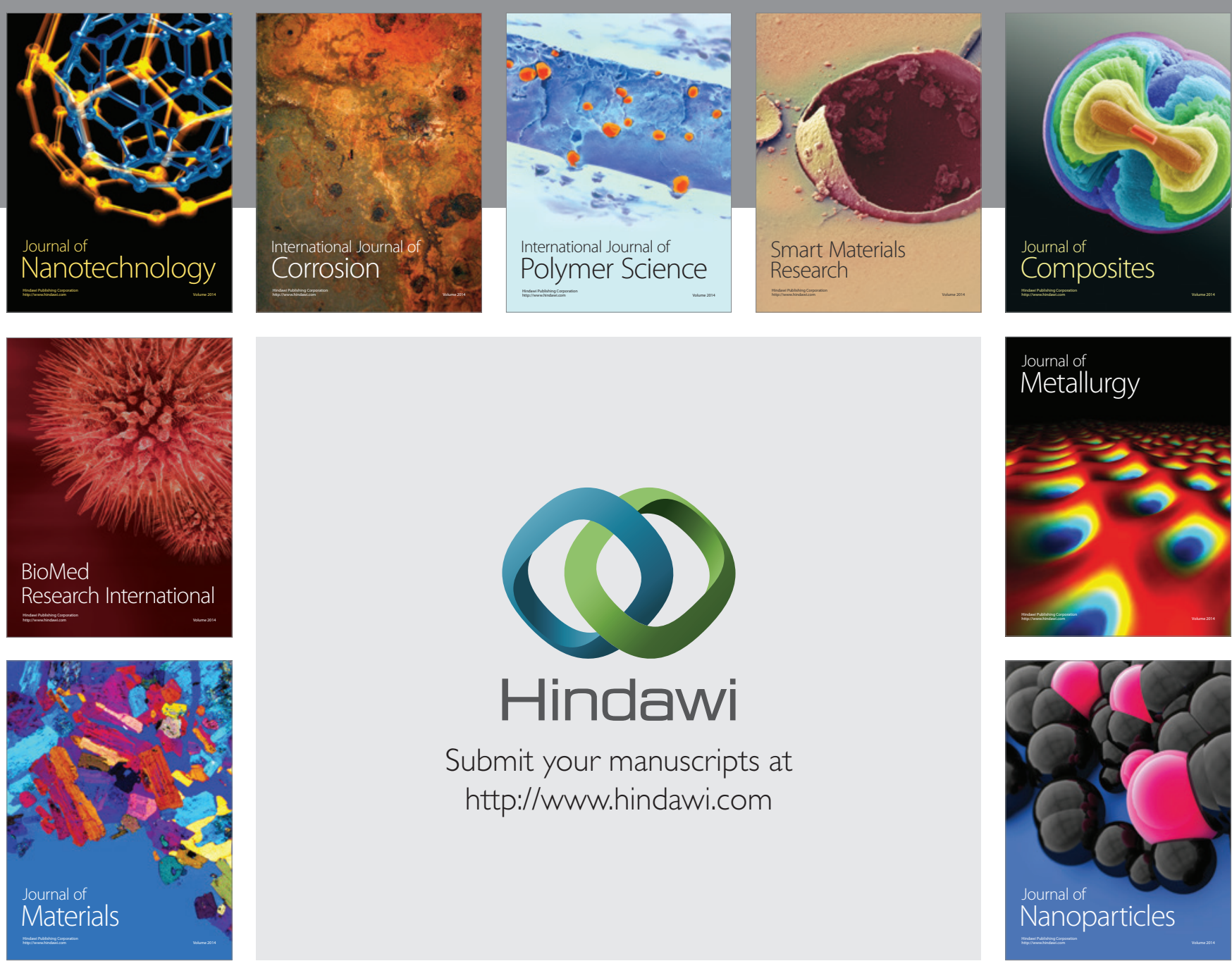

\section{Hindawi}

Submit your manuscripts at

http://www.hindawi.com

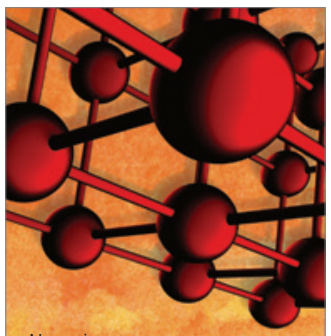

Materials Science and Engineering
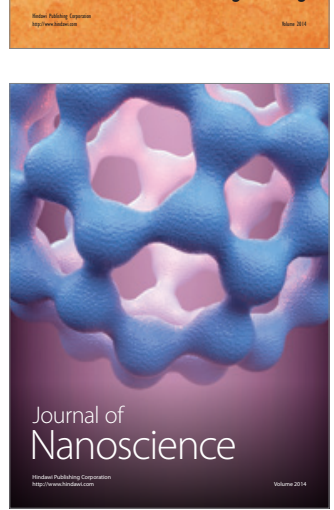
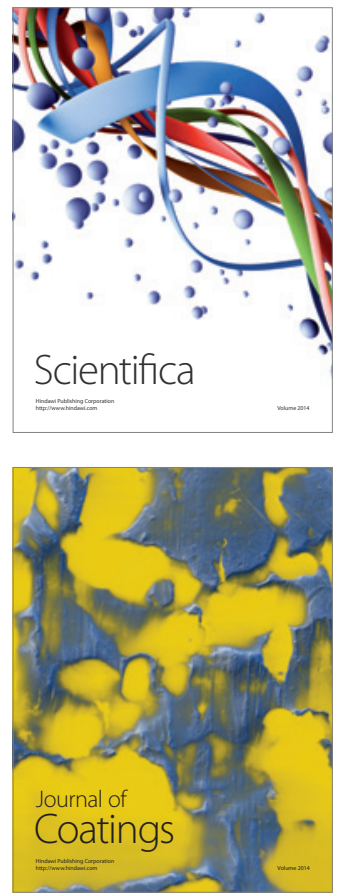
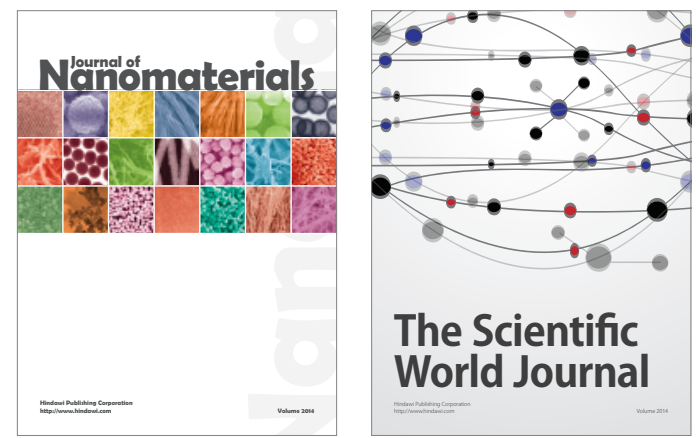

The Scientific World Journal
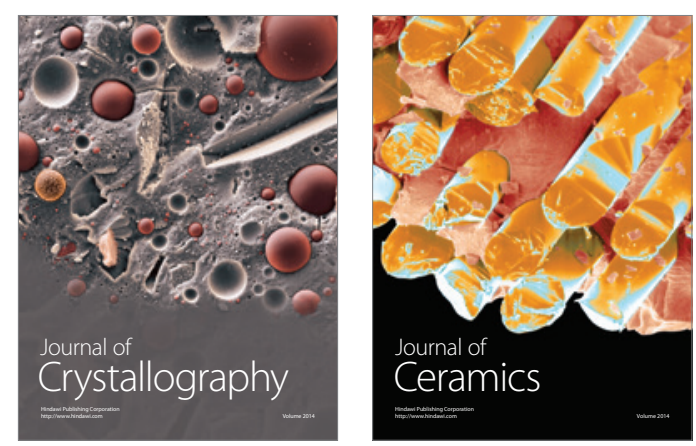
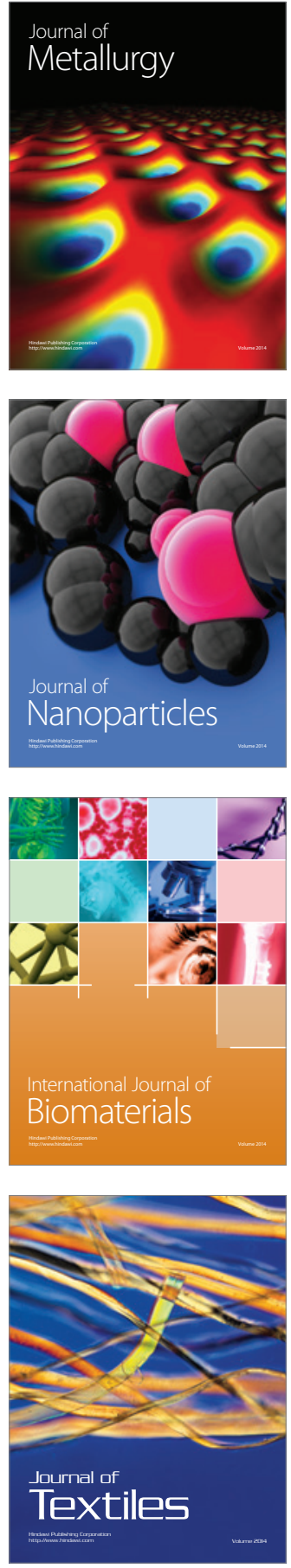